\title{
Lateral inhibition between spatially adjacent spatial-frequency channels?
}

\author{
DOV SAGI and SHAUL HOCHSTEIN \\ Institute of Life Sciences, Hebrew University, Jerusalem, Israel
}

\begin{abstract}
A new lateral inhibitory phenomenon is suggested between spatially neighboring channels that detect similar spatial frequencies. This mechanism tends to enhance the apparent contrast of gratings at their boundaries, and Mach-band-type effects are demonstrated at the edge of a suprathreshold half-field grating. We studied the dependence of this effect on the grating parameters of spatial frequency and contrast. The grating spatial frequency must be above about 4 cycles per degree (cpd) for any effect to be seen. In the range 4-14 cpd, the type of effect seen depends on the contrast of the grating: At high contrasts, the effect is an enhancement of grating contrast near its border, whereas at near threshold contrasts, an opposite effect, edge contrast diminution, is seen. Finally, above about $14 \mathrm{cpd}$, enhancement is found down to threshold contrasts. The latter very-high-frequency effect may be due to a high-spatial-frequency limit of the visual channels available, with the smallest receptive-field summation area being in the order of $2.2^{\prime}$. The near-threshold edge contrast diminution may derive from illumination lateral inhibition. In the intermediate range, however, the enhancement appears only at high contrast levels and therefore cannot be due to a linear mechanism. This enhancement seems to derive from lateral inhibition between neighboring spatial-frequency channels. A Craik-O'BrienCornsweet type of visual illusion is demonstrated in the spatial-frequency grating contrast domain. The effect of lateral inhibition on the selectivity of spatial frequency channels is discussed. Lateral inhibition may cause a shift in the optimal spatial frequency of the channels by about $40 \%$ at high contrasts, making such gratings appear to have lower spatial frequencies. This shift may also be responsible for the phenomenon of contrast constancy.
\end{abstract}

A central principle in the processing of information by the visual system is the enhancement of stimulus boundaries by lateral inhibition. Ernst Mach, noting the luminosity bands that today bear his name, inferred the existence of an underlying mechanism that performed lateral inhibition (Mach, 1865/1965). Mach bands are the bright and dark stripes seen along the edges of a sloped change in intensity of illumination. Their physiological counterpart has been studied in detail (Ratliff, 1965).

The accepted explanation for these effects is that neighboring detectors inhibit one another. Thus, a detector might not respond vigorously if its neighbors see the same intensity of light as it does. It will respond robustly only if at least some of its neighbors see less light than it does and thus have a limited inhibitory effect on it. Strong responses are, therefore, present only at illumination borders and not in the midst of areas of uniform illumination. The visual system must therefore infer the intensity of illumination upon an area, not from the absolute response of detectors whose gaze is centered on it, but rather from the relative information gained from detectors that see the height of the steps that lead up to it.

Lateral inhibition has been found to play a role in the

We thank Professor Peter Hillman for his helpful discussions and review of this paper. This study was supported by a grant from the Israel Academy of Sciences and Humanities.

D. Sagi's present mailing address is: Department of Applied Mathematics, The Weizmann Institute of Science, Rehovot 76 100, Israel. processing of other visual dimensions as well. Similar effects, or illusions, have been seen for the dimensions of grating orientation (Blakemore, Carpenter, \& Georgeson, 1970), spatial frequency (Mackay, 1973), line length (Crovitz, 1976), and depth (judged by binocular disparity; Anstis, Howard, \& Rogers, 1978), as well as velocity (Walker \& Powell, 1974).

The purpose of this research was to study the possible existence of a new kind of lateral inhibition between spatially neighboring channels that detect similar spatial frequencies. We demonstrate an enhancement of the apparent contrast of gratings at their boundaries. We explore the spatial frequency range of this phenomenon and study its contrast dependence. These effects may indeed derive from lateral inhibition between spatial frequency detectors.

In the course of previous studies on interactions between spatial frequency channels when performing tasks involving the discrimination of changes in contrast of suprathreshold compound gratings, it was found that highspatial-frequency channels show superlinearities in their response dependence on contrast (Sagi \& Hochstein, 1983, 1984). Areas of high local contrast are given disproportionate weight compared with other areas in contrast-discrimination tasks. The fact that this nonlinearity was found only at high spatial frequencies, above $4 \mathrm{cy}$ cles per degree (cpd), suggested that there might be shortrange lateral inhibitory interactions between neighboring channels. This finding led to the present study of the ex- 
istence and parameters of lateral inhibition between spatially adjacent high-spatial-frequency channels.

Barlow (1965) and Campbell, Carpenter, \& Levinson (1969) found that near threshold the visibility of the edgebar of a half-field grating is greater than that of the body of the grating itself. This edge enhancement seemed to us to be similar to the Mach-band effect one would expect at the border of a grating. However, this effect had been studied only at threshold contrasts, although lateral inhibitory effects would be expected to be even stronger at suprathreshold conditions (see Ratliff, 1965). Furthermore, Campbell et al. (1969) found the enhancement effect only at very high spatial frequencies and were able to explain the effect on the basis of a linear (one-channel) model simply from the shape of the spatial modulation sensitivity function.

We explore here the suprathreshold spatial frequency and contrast dependencies of the enhancement effect (see Methods and Results) and show that a linear mechanism cannot be the source of this new phenomenon (Discussion). We demonstrate in the spatial frequency domain a Craik-O'Brien-Cornsweet type of visual illusion, generally understood to be related to the phenomenon of lateral inhibition. Finally, the implications of a lateral inhibitory mechanism are discussed (Discussion and Appendix).

\section{METHODS}

In order to test the spatial frequency range of the effect, a grating step was used. Figure 1 displays this stimulus pattern. The grating had a sinusoidal waveform with $12 \%$ contrast (difference between maximum and minimum intensities divided by their sum) and was adjacent to an area of uniform illumination of intensity equal to that of the mean intensity of the grating. (The figure shows a grating of much higher contrast than was used in the actual tests.) This pattern was produced on an oscilloscope CRT screen (P31 phosphor) using a TV-type raster and varying the $Z$-axis intensity with a waveform generator (Exact, 126C). The $10 \times 12 \mathrm{~cm}$ screen was viewed at a distance of $1.2 \mathrm{~m}$.

Spatial-frequency thresholds were measured by the method of adjustment. The subjects had control over the spatial frequency dial on the waveform generator. They could increase or decrease the spatial frequency of the pattern according to the experimenter's instructions, and were instructed to stop whenever the effect appeared or disappeared. Contrast thresholds were measured the same way, except that the subjects controlled the contrast dial on the waveform generator. Display was continuous in time and the subjects, although instructed to concentrate on the grating edge, were allowed to move their eyes freely.

The subjects were unpaid university staff, using natural viewing conditions, including corrective lenses as needed. The subjects (besides the authors) were not informed of the purpose of the study. Further details of the psychophysical procedure appear in each of the Results sections.

\section{RESULTS}

\section{The Spatial-Frequency Range of the Lateral Inhibitory Effect}

For a spatial-frequency-grating edge, a Mach band is equivalent to an increase in contrast of the first cycle(s) of the grating. Indeed, all subjects $(\mathrm{N}=8)$ reported that the first bright half-cycle of the grating appeared brighter than the other grating bars. (We shall refer to half-cycles of the grating as "bars," although the grating was sinusoidal in profile.) The reader may see this enhancement of the last half-cycle of the grating in Figure 1. Best viewing is from a distance of about $1 \mathrm{~m}$.

We found that the enhancement effect divides the spatial frequency domain into three ranges: At very high spatial frequencies, the enhancement is present right down to threshold levels; at intermediate spatial frequencies, the enhancement effect is only suprathreshold and reverses for low contrasts; and at very low spatial frequencies, no edge effect is seen at all. We first studied the lowfrequency cutoff of the effect.

The subjects were asked to reduce the spatial frequency of the grating (by adjusting the spatial-frequency-control dial on the waveform generator) while attending to the bright end bar. All subjects reported that the end bar finally became equal in intensity to the other bars. Alternatively, the subjects were asked to increase the spatial frequency of a grating whose end bar appeared to be of equal intensity as the other bars until it began to appear brighter than the other bars. The reader may approximately perform this task by slowly reducing the viewing distance while looking at Figure 1.

The subjects were then asked to find the lowest spatial frequency at which the bar was just brighter than the second bar. Eight subjects performed this task at least three times each. Their results are presented in Table 1 . The

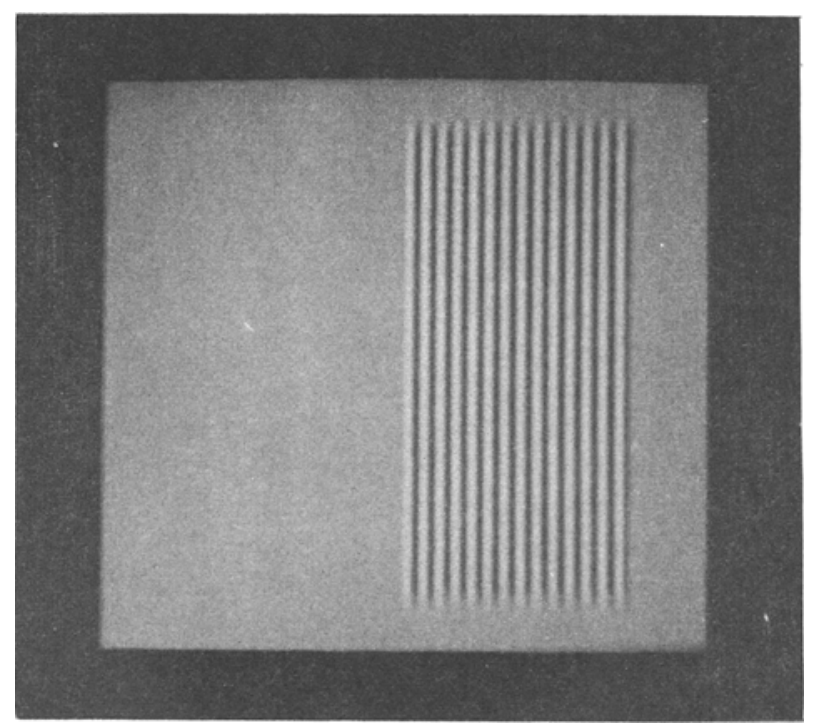

Figure 1. Enhancement of the last half-cycle of a grating-contrast step. The picture shows a grating step. The step between the highcontrast grating and the (zero-contrast) uniform illumination induces enhancement of the last bright half-cycle of the grating. This may be easily seen when the picture is viewed from a distance of at least $1 \mathrm{~m}$. Bringing the picture closer to the eye reduces the spatial frequency, and the enhancement effect disappears. 
Table 1

The Minimal Spatial Frequency for Suprathreshold Contrast-Step Enhancement

\begin{tabular}{cccc}
\hline Subject & $\begin{array}{c}\text { Average Minimum } \\
\text { Frequency (cpd) }\end{array}$ & SD & $\begin{array}{c}\text { Number of } \\
\text { Trials }\end{array}$ \\
\hline A.A. & 5.97 & .35 & 3 \\
P.A. & 5.76 & .31 & 5 \\
N.G. & 5.04 & .27 & 3 \\
P.G. & 2.64 & .50 & 4 \\
S.H. & 3.40 & .38 & 3 \\
D.S. & 3.84 & .37 & 9 \\
H.S. & 3.92 & .46 & 4 \\
M.S. & 3.80 & .75 & 6 \\
Average & 4.30 & 1.17 & \\
\hline
\end{tabular}

Note - The standard deviation for each subject is based on intertrial deviations; that for the average is based on intersubject deviations.

mean spatial frequency for this low-frequency cutoff was found to be $4.3 \mathrm{cpd}(\mathrm{SE}=0.41 \mathrm{cpd} ; \mathrm{N}=8$ ). This is the region of spatial frequencies where the human visual system is most sensitive. Note the small scatter of this result for the different subjects.

\section{The Contrast Dependence of the Effects}

The next study made use of the observation (see above) that, at low contrasts and intermediate frequencies, the end bar does not appear brighter than the other bars; in fact, it may appear less bright. Thus, at these frequencies, there is a range of near-threshold contrasts where the grating is visible but its end bar is invisible. The grating then appears to end with a dark half-cycle instead of with a bright half-cycle. However, at very high spatial frequencies, the end bar is visible and enhanced right down to threshold contrast levels. This half-cycle is therefore visible even when the rest of the grating goes below threshold and is invisible (Campbell et al., 1969). The dependence of the effect on grating contrast (in the intermediate spatial-frequency range), showing enhancement for high-contrast gratings and diminution for low-contrast gratings, clearly indicates that the source of the effect cannot be a linear processing mechanism. The spatial frequency that divided the medium- and high-spatial-frequency ranges was now determined.

The subjects were asked to reduce the contrast of grating patterns similar to that of Figure 1 (by adjusting the contrast dial on the waveform generator) down to threshold and to report whether the grating or the bar disappeared first. Alternatively, the subject increased the contrast of the grating from below threshold and reported whether the grating or the bar appeared first. From a series of such tasks, using a set of different spatial frequencies, the dividing line between the medium- and the high-spatial-frequency ranges was determined for each subject. Table 2 presents the results for four subjects. The mean spatial frequency was $13.6 \mathrm{cpd}(\mathrm{SE}=1.7 \mathrm{cpd} ; \mathrm{N}=4)$. For gratings above this frequency, the enhancement effect appears even at subthreshold levels; below this spatial frequency, there is a range of contrasts near threshold where the grating is visible but the end bright half-cycle is not.

\section{DISCUSSION}

The purpose of this paper was to establish the existence of the spatial-frequency-channel lateral inhibitory effect and to find the spatial-frequency range of the effect. The existence of the effect is most clearly demonstrated by the very ability of the subjects to carry out reproducibly the quantitative parametric tasks set them. In addition, the reader may see for him- or herself the effects in the figures.

Is lateral inhibition responsible for the enhancement effect seen here? And, if so, is it responsible for the supraand/or the subthreshold effects seen in the different spatialfrequency ranges? We shall suggest that lateral inhibition is responsible for the medium spatial-frequency-range suprathreshold enhancement effect, but that it may not be responsible for the very high-spatial-frequency subthreshold phenomenon previously studied by Campbell et al. (1969).

The enhancement of the end "bar' of the grating, as in Figure 1, cannot be due to illumination lateral inhibition, which is responsible for the usual contrast and Mach band phenomena. This is because illumination lateral inhibition would tend to make this bar appear less bright, rather than enhanced, since the uniform illumination on one of its sides is brighter than the dark bars surrounding the other bright bars. Thus, the phenomenon seen cannot be due to lateral inhibition between illumination detectors.

An alternative mechanism, which would lead to endbar enhancement would be a receptive field that simply summed the illumination over a rather broad area. If such a field were broad enough, it would be less stimulated when positioned over a number of cycles of the grating than when positioned over the end bright half-cycle and the area of uniform illumination adjacent to it. Thus, out of a series of such illumination-summing detectors, the biggest response would be that of the detector covering just the end bar and part of the adjacent uniform illumination area-an enhancement effect. Is it reasonable to expect that such an illumination-summing mechanism can be responsible for the end bar enhancement reported here? Previous studies of this phenomenon may shed light on this question:

Barlow (1965) showed that a moving grating might be detected above the diffraction limit by means of the flicker that appears at the edge of the aperture as each bar is occluded. He pointed out that, for stationary gratings slightly

Table 2

The Minimal Spatial Frequency for Subthreshold Enhancement of a Contrast Step

\begin{tabular}{|c|c|c|}
\hline Subject & $\begin{array}{c}\text { Minimum } \\
\text { Frequency (cpd) }\end{array}$ & SD \\
\hline $\begin{array}{l}\text { M.S. } \\
\text { H.S. } \\
\text { S.H. } \\
\text { D.S. }\end{array}$ & $\begin{array}{r}13.2 \\
13.5 \\
9.8 \\
17.9\end{array}$ & \\
\hline Average & 13.6 & 3.32 \\
\hline
\end{tabular}


below the ordinary resolution limit, the bar near the edge could be correctly seen as dark or light.

Campbell et al. (1969) studied the threshold detectability of half-field gratings at high spatial frequencies ( $>20 \mathrm{cpd}$ ) where the edge bar of the grating is visible although the body of the grating is not. They showed that the contrast sensitivity of a half-field grating is slightly below half that of a single grating bar.

Macleod and Rosenfeld (1972) analyzed the data of Campbell et al. (1969) with three space-domain models. They noted that if one employs the maximum responses across the range of field sizes (or channel spatial frequencies) and across the possible translations of the fields over the stimuli (assuming local channels), then the predicted contrast sensitivities of a half-field grating should always be at least as high as that of a full sine-wave grating. Since the half-field grating data of Campbell et al. (1969) show sensitivities that are lower than that of a full grating in the intermediate frequency range $(6-20 \mathrm{cpd})$, these observers probably adopted criteria involving the visibility of detail in the vicinity of the edge of the grating rather than over the entire stimulation field.

When the sensitivity versus field size (or spatial frequency) function is relatively constant, the contrast sensitivity of the light bar at the edge of the half-field grating is approximately $70 \%$ of the sensitivity of a bar in the interior of the grating, as a result of the lack of a dark bar in one of the inhibitory zones flanking the receptive field. Thus, an observer who restricts his or her judgments to the visibility of the edge bar of a half-field grating should show sensitivities that are lower than those he or she would show when tested for visibility of a full grating - in the intermediate frequency range. This was, indeed, the case for Campbell et al.'s (1969) observers. In no case was there a sensitivity to a half-field grating in the intermediate range (6-20 cpd), which was greater than that for a full-field grating.

Extending these findings to the suprathreshold range, we would expect the interior of a half-field grating to appear to have higher contrast than the edge bar. Just the opposite effect is seen, and the explanation of this effect cannot derive from the linear predictions of the singlechannel (Campbell et al., 1969) or the multiple-channel (Macleod \& Rosenfeld, 1972) models.

We make the assumption that, of the available channels, the visual system would use those most suited for detecting any particular stimulus profile. Thus, a grating would be detected mainly by the channel best tuned to its spatial frequency and not by a channel with a considerably broader receptive-field illumination-summing area, unless the broader field channel was considerably more sensitive than the higher frequency, better tuned channel. The only case in which the visual system would use too broad a channel would be for stimulation with gratings of such high spatial frequency that the best available channels would be the highest spatial-frequency channels of the system. Thus, above $13.7 \mathrm{cpd}$, it may indeed be possible that the enhancement seen, which is present down to subthreshold contrasts, derives in part from the system's using larger receptive fields than appropriate for the stimulus grating pattern. This would mean that the highest spatial-frequency channels would be of the order of $13.7 \mathrm{cpd}$, corresponding to a receptive-field excitatory summation area of $2.2^{\prime}$. Wilson and Bergen (1979) estimate, on the basis of threshold measurements, the smallest fields to be of 3'. Marr, Poggio, and Hildreth (1980) estimate, on the basis of theoretical considerations and visual hyperacuity, that there may be fields as small as $1.3^{\prime}$. Similarly, cat and monkey cortical electrophysiological measurements show receptive fields of sizes down to 2' -4 ' (Poggio, Doty, \& Talbot, 1977). Thus, it is entirely reasonable to assume that enhancement in the highspatial-frequency range is due, at least in part, to the use for this task, in this spatial-frequency range, of oversized receptive fields. This mechanism would be a linear one and would extend down to threshold and even subthreshold contrast levels, as found by Campbell et al. (1969) and confirmed here.

A similar summation effect could be caused by optical blurring due to light scatter by the pupil and lens. A minimal spot of light would be spread to a circle of diameter 1'-2', depending on light conditions (Campbell \& Green, 1965; Westheimer, 1963). This optical mechanism would also be linear and extend down to subthreshold levels. The results presented here can neither reject these two possible sources of the very high-spatial-frequency enhancement phenomenon nor differentiate between them.

The intermediate-spatial-frequency, suprathreshold enhancement cannot be explained by these mechanisms, however, and seems instead to be caused by lateral inhibition between spatially neighboring channels of similar frequencies. The very presence of two enhancement forms, subthreshold and suprathreshold, at two separate spatial-frequency ranges supports the idea of two underlying mechanisms. The lateral inhibition would have a threshold somewhat higher than that of detection by each channel (cf. Ratliff, 1965). This inhibition is therefore not a linear function of contrast, corresponding to the finding that greater weight is given to local areas of high contrast in the intermediate and high-spatial-frequency ranges (Sagi \& Hochstein, 1983, 1984). Note that the inhibition may be spatial-frequency specific or may be broadband. The suggestion that it may be between channels of similar frequencies is only the simplest hypothesis, and is testable.

Physiological correlates' have been found for illumination lateral inhibition. Have similar studies presented evidence to support the presence of spatial frequency contrast inhibition? Maffei and Florentini (1972) have suggested that lateral geniculate nucleus receptive-field surrounds may derive from retinal ganglion cell centers, which themselves have inhibitory surrounds. These lateral geniculate receptive fields would then constitute detectors with lateral inhibition from fields that are spatially adjacent and have similar spatial-frequency tuning. Alternatively, the effect could be cortical in origin. If the 
underlying mechanism were in the primary visual cortex, one could explain the lack of an enhancement effect for gratings of spatial frequency below $4 \mathrm{cpd}$, since most cortical short-range interactions might be expected to be within a single hypercolumn of a width of about $10^{\prime}-15^{\prime}$ (Hubel \& Wiesel, 1968).

Complementary to the Mach band phenomenon is the well-known Craik-O'Brien-Cornsweet visual illusion, in which sharp and gradual changes in illumination are joined to produce the appearance of an illumination step where none exists. The visual system disregards the slow illumination gradient, enhances the faster change, and concludes that a step in intensity is present (see O'Brien, 1958).

A similar phenomenon in the spatial-frequency domain is demonstrated in Figure 2. The two sides of the figure appear to have different contrasts. A Craik-O'Brien-Cornsweet type of contrast edge is interspaced between the two halves, but the contrasts far from the boundary are, in fact, identical. There is a sharp increase in contrast superposed on a gradual decrease in contrast going from left to right. The reader should examine the figure from at least 1-m away for best effect. The apparent difference in contrast may be canceled by covering, with a strip of gray paper, the central six stripes of the figure where the two opponent contrast changes occur.

\section{The Effect of Lateral Inhibition on the Selectivity of Spatial-Frequency Channels}

Lateral inhibitory interactions between neighboring channels may affect their spatial-frequency selectivity characteristics. For example, photoreceptors are very broadband spatial-frequency detectors. Lateral inhibition between such receptors reduces their spatial-frequency bandwidth by introducing low-frequency attenuation. The effect of lateral inhibition (as in the retinal ganglion cell receptive field) may be seen mathematically as a second derivative operator $\left(-\partial^{2} / \partial x^{2}\right)$. In particular, such a field will not respond to uniform or to uniformly graded illumination stimuli, for which the second derivative is zero. As discussed in the Appendix, after Fourier transformation, in the spatial frequency (f) domain, the second derivative operator becomes equivalent to multiplication by $\mathrm{f}^{2}$.

Thus, each stage of lateral inhibition, translated to the second derivative operator, becomes, in the spatial frequency domain, simply a stage of multiplication by $\mathrm{f}^{2}$. Figure 3 displays the spatial-frequency sensitivity profiles $S_{1}, S_{2}, S_{3}$, demonstrating the effects of the introduction of successive stages of lateral inhibition, as follows: $S_{1}$ is a first-order luminosity detector (photoreceptor) with a gaussian sensitivity profile. $S_{2}$ is a receptive field with antagonistic center and surround areas-that is, displaying luminance lateral inhibition. The response of $S_{2}$ is the second derivative with respect to position of the response of $S_{1}$, or equivalently the response of $S_{1}$ multiplied by $f^{2}$. The addition of a further stage of lateral inhibition, as proposed in this report, leads to $S_{3}$, whose response is that of $S_{2}$ multiplied by an additional factor of $f^{2}$.
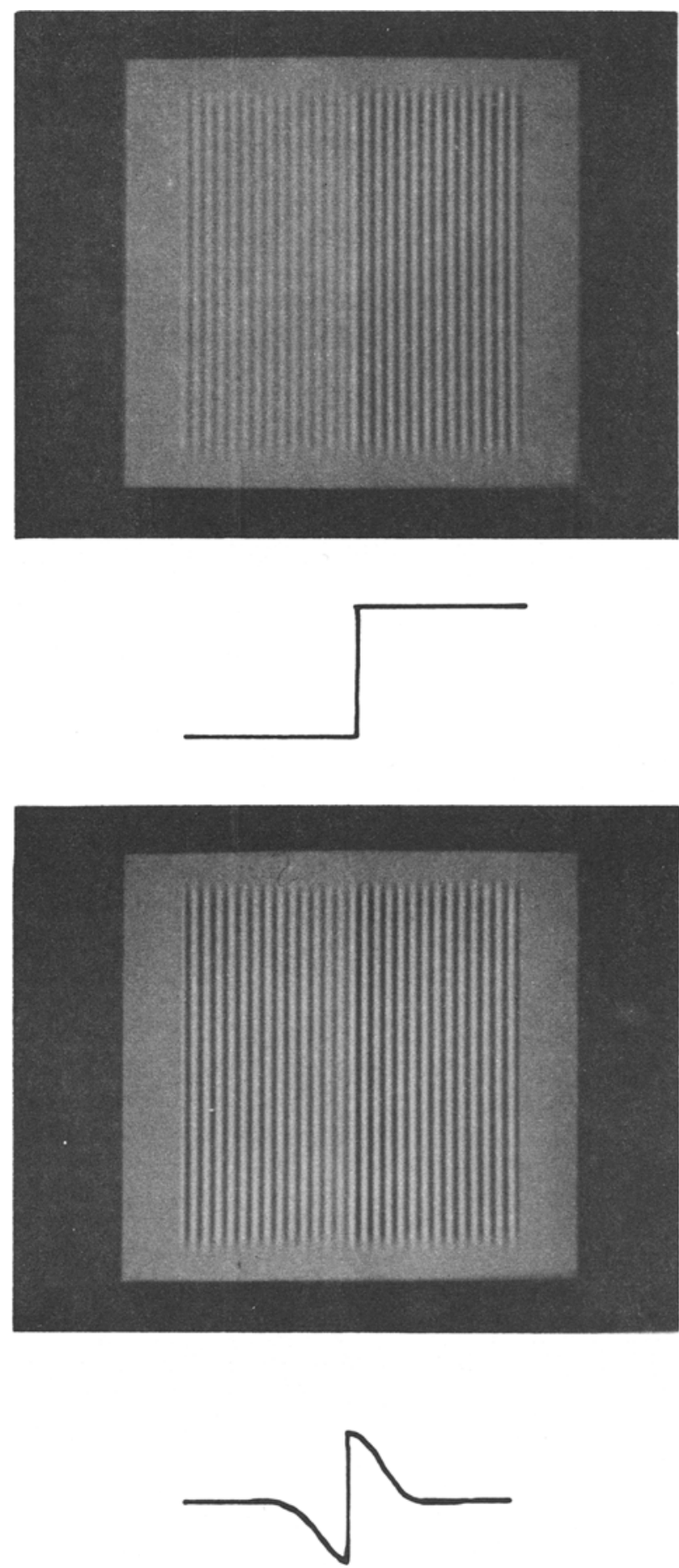

Figure 2. The Craik-O'Brien-Cornsweet type of contrast visual illusion. The bottom picture's grating appears to have greater contrast on the right than on the left, as is, in fact, the case for the top picture's grating. In fact, a rapid change in contrast is superposed on a gradual change in the opposite direction. Covering the central strip of the picture makes the illusion disappear and the two sides of the picture to appear to have identical contrast, as indeed they do. This illusion is probably due to lateral inhibition between spatial-frequency detectors. The line graphs indicate the grating contrast as a function of position along the pictures. The illusion is best seen when viewed from at least $1 \mathrm{~m}$. 


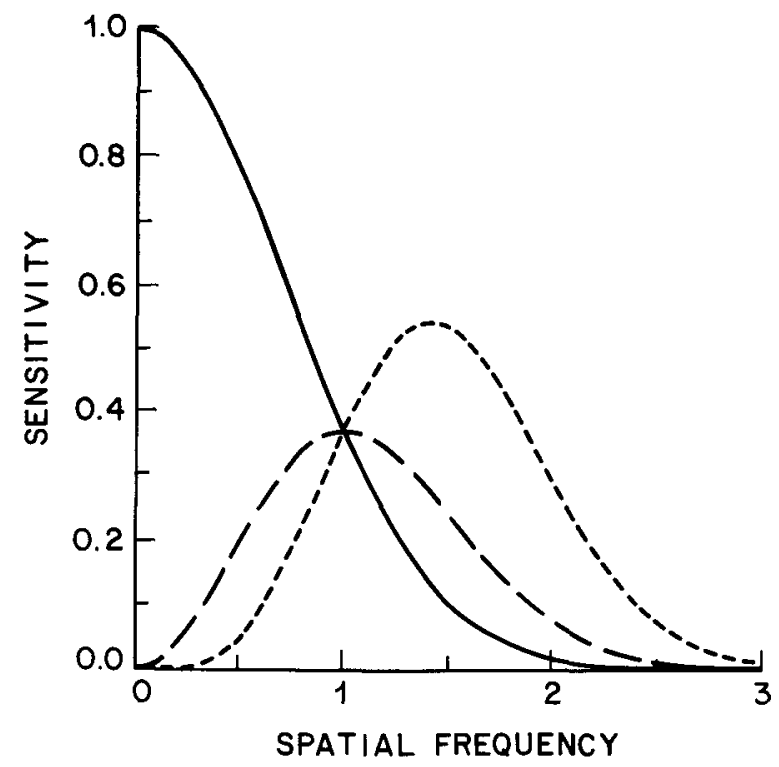

Figure 3. The theoretical spatial-frequency sensitivity profiles of three stages of visual units. The second and third stages include luminance and spatial-frequency lateral inhibition, respectively. The first stage (photoreceptors) has a gaussian sensitivity profile, peaking at zero frequencey (DC). The second has a peak sensitivity at $f_{0}$ and a DOG (difference-of-gaussians) shape. The third has a peak at $1.4 f_{0}$ due to lateral inhibition between neighboring spatialfrequency channels.

What are the spatial-frequency sensitivities of these profiles? $S_{1}$ is maximally sensitive to low spatial frequencies, down to uniform fields (DC). At high spatial frequencies, the sensitivity $S_{1}$ drops to zero. At the frequency $f_{0}$, the sensitivity, $S_{1}$, is at $1 / e$ height. The second-stage receptive field, $S_{2}$, has a low-frequency attenuation, as well. Its maximal sensitivity is at spatial frequency $f_{0}$. This field, with antagonistic center and surround, each with a gaussian profile, may be described as a difference-of-gaussians (DOG-Marr \& Hildreth, 1980; Wilson \& Bergen, 1979); it has a full bandwidth at half-height of about 1.8 octaves.

The third stage, $S_{3}$, is similar to $S_{2}$ with a factor $\mathrm{f}^{4}$ instead of $f^{2}$, which causes a shift in the position of the maximal sensitivity by $40 \%$ (see Appendix).

If the lateral inhibition is contrast dependent, as demonstrated in our results, then the spatial frequency of the optimal sensitivity will also depend on contrast. At low contrasts, close to threshold, where there is no lateral inhibition, and hence no spatial frequency shift, the maximal sensitivity will be at spatial frequency $f_{0}$. At high contrasts, where the lateral inhibition is maximal, the optimal sensitivity will be at a frequency that is higher than $f_{0}$ by $40 \%$. Georgeson (1980) has, indeed, described a shift in the apparent spatial frequency of a grating with the increase in the contrast of the stimulation. As the contrast of a grating with fixed spatial frequency is increased, the frequency of the grating appears to decrease. Georgeson suggests that "the perceptual changes occur because each channel carries an invariant perceptual 'sign' or meaning that does not alter when the spatial selectivity of the channel shifts." The visual system recognizes each neuron as representing a certain spatial frequency. This representation, or label, is determined by the optimal frequency of the unit at low contrasts. As the contrast is increased, the selectivity of the unit to its "optimal" frequency is reduced while the sensitivity of another unit, labeled to represent a lower frequency, becomes higher for this frequency. Thus, high-contrast gratings of a particular frequency will be detected mainly by units that are labeled as representing lower spatial frequencies, and will "appear" to have lower frequencies. The maximal apparent spatial-frequency shift reported by Georgeson (1980) was $30 \%-40 \%$, matching the shift calculated on the basis of lateral inhibition. This coincidence supports the hypothesis that the mechanism underlying the spatial frequency shift found by Georgeson is indeed lateral inhibition.

A shift of the visual system toward higher spatial frequencies may also be responsible for the contrast constancy phenomenon (Georgeson \& Sullivan, 1975). The apparent contrast of intermediate frequencies rises gradually with stimulus contrast, whereas the apparent contrast of high spatial frequencies rises more rapidly as lateral inhibition shifts the most sensitive channels from the intermediate- towards the high-spatial-frequency range. This phenomenon has not been studied physiologically, although Bisti, Clement, Maffei, and Mecacci (1977) and Movshon, Thompson, and Tolhurst (1978) found a few cat cortical units sensitive to high spatial frequencies whose optimal spatial frequency rose with contrast.

\section{CONCLUSION}

The existence, characteristics, and implications of a new phenomenon-lateral inhibition between spatial-frequency channels whose receptive fields are spatially adjacent-have been demonstrated and analyzed. This inhibition has a nonlinear dependence on stimulus contrast and is not present at low spatial frequencies ( $<4 \mathrm{cpd}$ ). At very high frequencies ( $>14 \mathrm{cpd}$ ), another effect is introducedthe high-frequency cutoff of the available channels. The main effect of the lateral inhibition is to enhance the appearance of changes in contrast, giving special prominence to steps of contrast change in space. This enhancement is nonlinear. It is not present for very low contrasts, allowing the system to detect the presence of a grating with very low contrast over a wide spatial area. An additional implication of the lateral inhibition between neighboring spatial frequency channels is that it tends to shift the spatial-frequency sensitivity range of each channel towards higher spatial frequencies and to sharpen the tuning of the channels. This is analogous to the effect of brightness lateral inhibition. The nonlinearity means that these effects are strengthened with increasing stimulus contrast so that high-contrast pictures may have sharp fine details. 


\section{REFERENCES}

Anstis, S. M., Howard, I. P., \& Rogers, B. (1978). A Craik-O'BrienCornsweet illusion for visual depth. Vision Research, 18, 213-217.

BARLOW, H. B. (1965). Visual resolution and the diffraction limit. Science, 149, 553-555.

Bisti, S., Clement, R., Maffei, L., \& Mecacci, L. (1977). Spatial frequency and orientation tuning curves of visual neurones in the cat: Effects of mean luminance. Experimental Brain Research, 27, 335-345.

Blakemore, C., Carpenter, R. H. S., \& Georgeson, M. A. (1970). Lateral inhibition between orientation detectors in the human visual system. Nature, 228, 37-39.

Campbell, F. W., Carpenter, R. H. S., \& Levinson, J. Z. (1969). Visibility of aperiodic patterns compared with that of sinusoidal gratings. Journal of Physiology (London), 204, 283-298.

Campbell, F. W., \& Green, D. G. (1965). Optical and retinal factors affecting visual resolution. Journal of Physiology (London), 181, 576-593.

Crovitz, H. F. (1976). Perceived length and the Craik-O'Brien illusion. Vision Research, 16, 435.

Georgeson, M. A. (1980). Spatial frequency analysis in early visual processing. Philosophical Transactions of the Royal Society, London, Series $B, 290,11-22$.

GeORGESON, M. A., \& Sullivan, G. D. (1975). Contrast constancy: Deblurring in human vision by spatial frequency channels. Journal of Physiology (London), 252, 627-656.

HUBEL, D. H., \& WIESEL, T. N. (1968). Receptive fields and functional architecture of monkey striate cortex. Journal of Physiology (London), 195, 215-243.

MACH, E. (1865). Über die Wirkung der räumlichen Vertheilung des Lichtreizes auf die Netzhaut. Sitzungsberichte der mathematischnaturwissenschaftlichen Klasse der Kaiserlichen Akademie der Wissenschaften, 5, 303-322. [Parts translated as an appendix in Ratliff, F. (1965). Mach bands: Quantitative studies on neural networks in the retina. San Francisco: Holden-Day.]

MACKAY, D. M. (1973). Lateral interactions between neural channels sensitive to texture density. Nature, 245, 159-161.

MACLEOD, I. D. G., RosenfeLD, A. (1972). The visibility of aperiodic bar patterns: Predictions of a space-domain model (Technical Report TR-209). College Park: University of Maryland, Computer Science Center.

MAFFEI, L., FloRentinI, A. (1972). Retinogeniculate convergence and analysis of contrast. Journal of Neurophysiology, 35, 65-72.

MARR, D., \& Hildreth, E. (1980). Theory of edge detection. Proceedings of the Royal Society, London, Series B, 207, 187-217.

MARR, D., Poggio, T., \& Hildreth, E. (1980). Smallest channel in early human vision. Joumal of the Optical Society of America, 70, 868-870.

Movshon, J. A., Thompson, I. D., \& Tolhurst, D. (1978). Spatial and temporal contrast sensitivity of neurons in areas 17 and 18 of the cat's visual cortex. Jourmal of Physiology (London), 283, 101-120.

O'Brien, V. J. (1958). Contour perception, illusion and reality. Journal of the Optical Society of America, 48, 112-119.

Poggio, G. F., Doty, R. W., \& TALBot, W. H. (1977). Foveal striate cortex of behaving monkey: Single-neuron responses to square wave grating during fixation of gaze. Journal of Neurophysiology, 40, 1369-1391.

RATLIFF, F. (1965). Mach bands: Quantitative studies on neural networks in the retina. San Francisco: Holden-Day.

SAGI, D., \& HochSTEIN, S. (1983). Discriminability of supra-threshold compound spatial frequency gratings. Vision Research, 23, 1595-1606.

SAGI, D., \& HochSTEIN, S. (1984). The contrast dependence of spatial frequency channel interactions. Vision Research, 24, 1357-1365.

WALKER, P., PowelL, D. J. (1974). Lateral interaction between neural channels sensitive to velocity in the human visual system. Nature, 252, 732-733.

WESTHEIMER, G. (1963). Optical and motor factors in the formation of the retinal image. Journal of the Optical Society of America, 53, 86-93.

WILSON, H. R., \& BERGEN, J. R. (1979). A four mechanism model for threshold spatial vision. Vision Research, 19, 19-32.

\section{APPENDIX}

The Effect of Lateral Inhibition on Spatial

Frequency Channel Bandwidth and Frequency of Maximal Sensitivity

Assume first-order luminosity detectors (photoreceptors) with a gaussian spatial sensitivity profile. Such receptors will have a gaussian sensitivity profile also in the spatial-frequency domain with a peak at zero,

$$
\mathrm{S}_{1}(\mathrm{f})=\mathrm{Ce}^{-\left(\mathrm{f} / \mathrm{f}_{0}\right)^{2}}
$$

This sensitivity profile is shown in Figure 3. It has its peak at zero spatial frequency (corresponding to uniform illumination) and falls to $1 / \mathrm{e}$ amplitude at $f_{0}$. The response of such a gaussian detector to a sinusoidal grating of spatial frequency $f$ and luminance profile $\sin (\mathrm{fx})$ will be

$$
R_{1}(f, x)=C \sin (f x) e^{-\left(f / f_{0}\right)^{2}} .
$$

Taking the second derivative of this response with respect to the position $\mathrm{x}$ produces the response expected from a receptive field with antagonistic center and surround areas-that is, displaying luminance lateral inhibition. The response of such a receptive field is

$$
R_{2}(f, x)=\frac{-\partial^{2} R_{1}(f, x)}{\partial x^{2}}=f^{2} C \sin (f x) e^{-\left(f / f_{0}\right)^{2}}
$$

so the spatial frequency sensitivity of the field may be defined as

$$
S_{2}(f)=f^{2} \mathrm{Ce}^{-\left(f / f_{0}\right)^{2}} .
$$

This sensitivity profile has its peak sensitivity at $f_{0}^{2}$ (see Figure 4 ). Lateral inhibition between such detectors, as proposed in this report, would have the effect of introducing another stage of second derivation of the response, namely,

$$
R_{3}(f, x)=\frac{-\partial^{2} R_{2}(f, x)}{\partial x^{2}}=f^{4} C \sin (f x) e^{-\left(f / f_{0}\right)^{2}}
$$

and a spatial frequency sensitivity profile of

$$
\mathrm{S}_{3}(\mathrm{f})=\mathrm{f}^{4} \mathrm{Ce}-\left(\mathrm{f} / \mathrm{f}_{\mathrm{o}}\right)^{2} .
$$

To find the sensitivity peak, we set the derivative with respect to frequency equal to zero:

$$
\begin{aligned}
\frac{\partial S_{3}(f)}{\partial f} & =\frac{\partial\left[\mathrm{f}^{4} \mathrm{Ce}^{\left.-\left(\mathrm{f} / \mathrm{f}_{0}\right)^{2}\right]}\right.}{\partial \mathrm{f}}=0 \\
f & =\sqrt{2} \mathrm{f}_{0}=1.4 \mathrm{f}_{0},
\end{aligned}
$$

a shift by $40 \%$ of the optimal spatial frequency as compared with the second stage. In general, this shift depends on the bandwidth of the channel of stage 2 , which was inherently assumed to be 1.8 octaves by the very use of the simplified form of the equations used above. In the next section, a more complex form is used in which the bandwidth is a free parameter, and it is shown that as the bandwidth is increased, the frequency shift is also increased. 


\section{The Shift in Channel Optimal Spatial Frequency} with Contrast as a Function of Channel Bandwidth

The shift in channel optimal spatial frequency is due to a change in the relative sensitivity of the channel over the range of spatial frequencies to which it is significantly sensitive. Thus, in order for the spatial frequency optimum to shift, the channel must be sensitive to additional spatial frequencies in the direction of the shift. If the channel bandwidth is too narrow, the channel will not respond to frequencies different from that of its optimum, and no shift can be induced.

Assume a channel with a gaussian spatial frequency sensitivity distribution, with a full bandwidth at half-height $b$, and optimal spatial frequency $f_{0}$. (A gaussian distribution is chosen, since it allows for the easiest analysis.) For $\left(f_{0} / b\right)>1$, this function has similar properties to those of the Fourier transform of a "difference-of-gaussians" (DOG, see above), and in particular a low spatial-frequency reduction in sensitivity:

$$
S_{2}(f)=C e^{\frac{-\left(\hat{f}-f_{e}\right)^{2}}{0.36 b^{2}}}
$$

As outlined above, application of lateral inhibition is equivalent to multiplication by $\mathrm{f}^{2}$, so that

$$
S_{3}(f)=f^{2} \mathrm{Ce}^{\frac{-\left(f-f_{0}\right)^{2}}{0.36 b^{2}}}
$$

To find the optimal spatial frequency, we set the derivative of $S_{3}(f)$ with respect to $f$ equal to zero:

$$
\frac{\partial S_{3}(f)}{\partial f}=2 f C e^{\frac{-\left(f-f_{0}\right)^{2}}{0.36 b^{2}}}-\left[\frac{\left(f-f_{0}\right)}{0.18 b^{2}}\right] \mathrm{f}^{2} \mathrm{Ce}^{\frac{-\left(f-f_{0}\right)^{2}}{0.36 b^{2}}}=0
$$

finding

$$
f^{2}-f f_{0}-0.36 b^{2}=0
$$

The solution to this quadratic equation is

$$
f=\frac{\left(f_{0}+\sqrt{f_{0}^{2}+1.44 b^{2}}\right.}{2}
$$

or

$$
\frac{\mathrm{f}}{\mathrm{f}_{0}}=\frac{1+\sqrt{1+1.44\left(\mathrm{~b}^{2} / \mathrm{f}_{0}^{2}\right)}}{2}
$$

The negative sign solution has been dropped because it represents an impossible condition-a negative spatial frequency. The positive-sign solution shows $f / f_{0}>1$ or a shift to higher spatial frequencies, with the degree of shift depending only on the bandwidth $\mathrm{b}$. In order to obtain a $40 \%$ shift in $\mathrm{f} / \mathrm{f}_{\mathrm{o}}$ (Georgeson, 1980 ), the above equation leads to $b / f_{0}=1.25$. Such a channel would have a bandwidth of 2.1 octaves at half height (see Discussion section in text). This is similar to the bandwidth of 2.2 octaves found by spatial frequency suprathreshold summation tests (see Sagi \& Hochstein, 1983, and references cited there). 